\title{
Characteristics of Nanoparticles-Based Chemical Sensors
}

\author{
Jason Stephens ${ }^{1}$, A. K. Batra ${ }^{1}$, James R. Currie $^{2}$ \\ ${ }^{1}$ Department of Physics (Materials Science Group), College of Engineering, Technology and Physical Sciences, Alabama A\&M \\ University, Normal, USA; ${ }^{2}$ NASA, George C. Marshall Space Flight Center, Huntsville, USA. \\ Email: ashok.batra@aamu.edu
}

Received February $16^{\text {th }}, 2012$; revised April $2^{\text {nd }}, 2012$; accepted May $8^{\text {th }}, 2012$

\begin{abstract}
Semiconducting nanoparticle tin oxide-based sensors have been prepared with a pressure load of 4, 6, 8, 10 tons and reinforced with carbon nanofibers $(\mathrm{CNF})$ in $\mathrm{SnO}_{2}$ matrix. The $\mathrm{SnO}_{2} / \mathrm{CNF}$ sensor's sensitivity for ethyl alcohol has increased by a factor of two, in compared with that of pure $\mathrm{SnO}_{2}$ 8-ton pressed sensor with lower response time. These results open the way towards further optimized lower cost CNF nanocomposite sensors as compared with expensive tin oxide/carbon nanotubes sensors.
\end{abstract}

Keywords: Chemical Sensors; Tin Oxide; Nano-Composites; Carbon Nanofiber

\section{Introduction}

Semiconductor metal oxides sensors are playing an important role in the detection of toxic pollutants $\left(\mathrm{CO}, \mathrm{H}_{2} \mathrm{~S}\right.$, $\mathrm{NO}_{\mathrm{x}}, \mathrm{SO}_{2}$, etc. $)$ and combustible gases $\left(\mathrm{H}_{2}, \mathrm{CH}_{4}\right.$ and flammable organic vapors, etc.) owing to their advantages of robust nature, less sensitive to moisture and temperature influence, simple electronic interface, quick response and recovery time [1]. Metal oxide materials such as $\mathrm{SnO}_{2}, \mathrm{ZnO}, \mathrm{TiO}_{2}, \mathrm{WO}_{2}, \mathrm{Ga}_{2} \mathrm{O}_{3}$, and others have been examined for gas sensing applications and for control of industrial processes. Tin oxide $\left(\mathrm{SnO}_{2}\right)$ is the most extensively studied material among other studied metal oxides [1,2]. Various techniques have been used to improve the sensitivity and selectivity of these sensors. As a matter of fact, a large part of the literature deals with characterization of sensors employing different forms of oxides, the effect of catalytic or other additives or ion implantation, use of masks and filters to improve selectivity and temperature programming techniques etc. [2]. However, a lack of consistency in sensor properties has been a major problem associated with various techniques used for the fabrication of sensors. Bulk, thick, and thin films of $\mathrm{SnO}_{2}$ have been used in the fabrication of gas sensors. Thin-film sensors are of great interest because of relatively small geometry, low power consumption, and sharp sensing effect, etc. A sensor having a thin film of less than a few hundred nanometers has a reasonably good sensitivity, but usually shows poor stability due to weak mechanical strength. The dispersions of dopants, which enhances, the sensitivity over thin film is not as satisfactory as those for thick or bulk type sensors [3-5].
For thick-film or bulk type sensor, dopants (or additives) are usually mixed homogeneously with the powder precursors. Recently, it has been reported that composite sensors incorporating differing proportions of tin oxide and zinc oxide exhibits higher sensitivity over a range of organic vapors [6]. Most importantly, the composite sensors have shown to have a significantly higher sensitivity than sensors fabricated from tin dioxide or zinc oxide, when operated under identical experimental conditions. It has been proposed that an increase in sensitivity is due to synergistic effects: complementary catalytic activity; and formation of hetero-junctions and changes in microstructure on sintering [7,8]. More recently, carbon nanotubes (CNTs) have been employed as active materials in semiconductor gas sensors [9-12]. The potential of CNTs of detecting gases arises from their very large surface area exceptional for electronic properties. However, due to their strong sp2 bonding in a near perfect hexagonal network, pristine carbon nanotubes are rather chemically inactive and this prevents the formation of chemical bonds with most molecules [10]. There are many factors affecting the gas-sensing properties. Besides, the intrinsic factors of materials, some extrinsic factors such as grain size, porosity and operating temperature, are also very important factors [13].

Our aim is to investigate bulk sensors via ceramic processing of nanoparticles of tin oxide fabricated under different pressing pressures, and reinforced with nanocarbon fiber composites for their response such as sensitivity and response time to ethyl alcohol. The study was also performed to determine the detection capability of sensors for different concentrations including their re- 
sponse mechanisms.

\section{Experimental}

\subsection{Sensor Fabrication}

Bulk Sensors were fabricated by optimized ceramic processing parameters. Tin (IV) oxide nanopowder (99.9\% metals basis, $50 \mathrm{~nm}$, MTI Corp.), and carbon nanofibers (diameter $150 \mathrm{~nm}$, surface are $20-30 \mathrm{~m}^{2} / \mathrm{gm}$, Pyrograph Products, Inc.) were used in the fabrication of the pellets. It is important to mention carbon nanotubes (CNT) and carbon nanofibers (CNF) are both hollow, nanometer in scale, and produced in a similar manner, there are distinct differences which significantly impact their performance and ability to be processed. The primary differences between the materials are morphology, size, ease of processing, and low-price. Carbon nanofibers, also known as Stacked-Cup Carbon Nanotubes (SCCNT), have a unique morphology in that graphene planes are canted from the fiber axis, resulting in exposed edge planes on the interior and exterior surfaces of the fiber. CNTs, on the other hand, typically resemble an assembly of concentric cylinders of grapheme. In each case, tin oxide mixtures were grounded and milled and the pressed under $4 \mathrm{~T}, 6 \mathrm{~T}, 8 \mathrm{~T}$ and $10 \mathrm{~T}$ to form pellets, thereafter named as ST4, ST6, ST8 and ST10 respectively. For the fabrication of nanocomposites, 0.01 gram of CNF were added to 2 grams of tin oxide mixtures and pressed under $8 \mathrm{~T}$ of pressure to form pellet of $14 \mathrm{~mm}$ diameter and $2 \mathrm{~mm}$ thickness, thereafter named as SCNF. The pellets were heated at the rate of $2^{\circ} \mathrm{C} / \mathrm{min}$ to $500^{\circ} \mathrm{C}$ and kept at this temperature for $2 \mathrm{~h}$, and then heated at the rate of $5^{\circ} \mathrm{C} / \mathrm{min}$ to a sintered temperature of $1000^{\circ} \mathrm{C}$ for $2 \mathrm{~h}$ in ambient air. Then pellets were cooled down to room temperature at the rate of approximately $1 \mathrm{C} / \mathrm{min}$. The summary of the processing steps of pellets are provided in Figure 1. Silver paste was used as contacts/electrodes on the same surface with a finite gap of about $3 \mathrm{~mm}$ as depicted in Figure 2.

\subsection{Vapor Measurement System}

The gas sensing properties were investigated under static conditions as illustrated in Figure 3 in a chamber of 5.2 liters [14]. The output resistance variations of the sensor were measured simultaneously with respect to temperature and time on injection of ethyl alcohol. The outputresistance was recorded using a Keithley 617 programmable electrometer connected to a computer. The resistance variation was measured for different concentrations of test vapors (ethyl alcohol) ranging from $50 \mu \mathrm{L}$ to 750 $\mu \mathrm{L}$ injected consecutively at appropriate time interval. The sensors were fixed onto a sample holder and the operating temperature was determined with a thermocouple attached to the sensor. Sensitivity was calculated using the following formula:

$$
\operatorname{Sensitivity}(S)=\frac{R_{a}}{R_{g}}
$$

where $R_{g}$ is the sensor resistance influenced by the ethyl alcohol vapors, and $R_{a}$ is the sensor resistance in the ambient air.

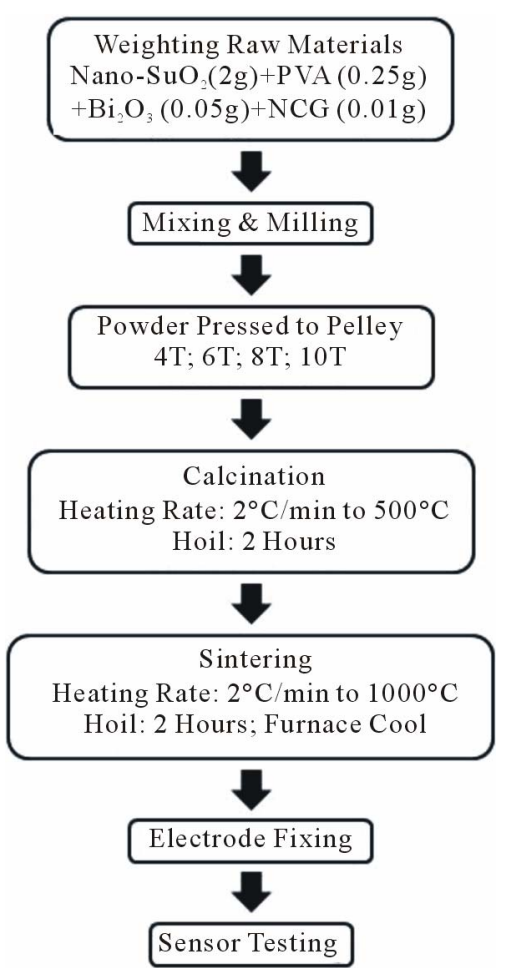

Figure 1. Process flowchart.

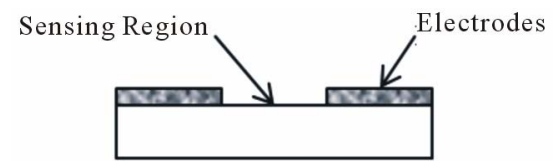

Figure 2. Fabricated chemical sensor design.

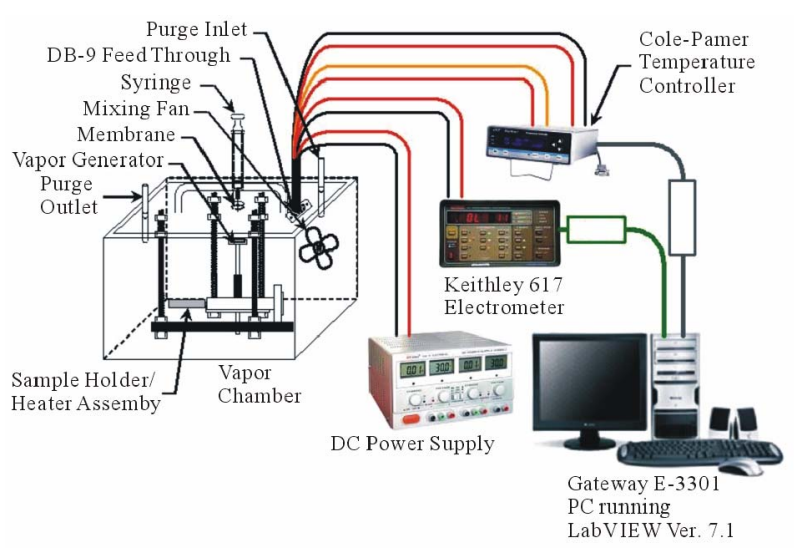

Figure 3. Static test fixture for evaluating chemical vapor response. 


\section{Results and Discussion}

In Figure 4 shows the response of the sensors with 50 $\mu \mathrm{L}$ of injected ethyl alcohol. Figures 5 and $\mathbf{6}$ illustrate the response as a function of ethyl alcohol concentration of ST8 and SCNF sensors. There is an increase of sensitivity with the increase of injected concentration of vapors. ST8 shows the highest sensitivity among the strongly pressed sensors. SCNF sensor shows the highest sensitivity among all sensors investigated. The sensitivity increases with concentration of injected ethyl alcohol. With a fixed surface area, a lower concentration of gas implies a lower coverage of gas molecules on the sur-

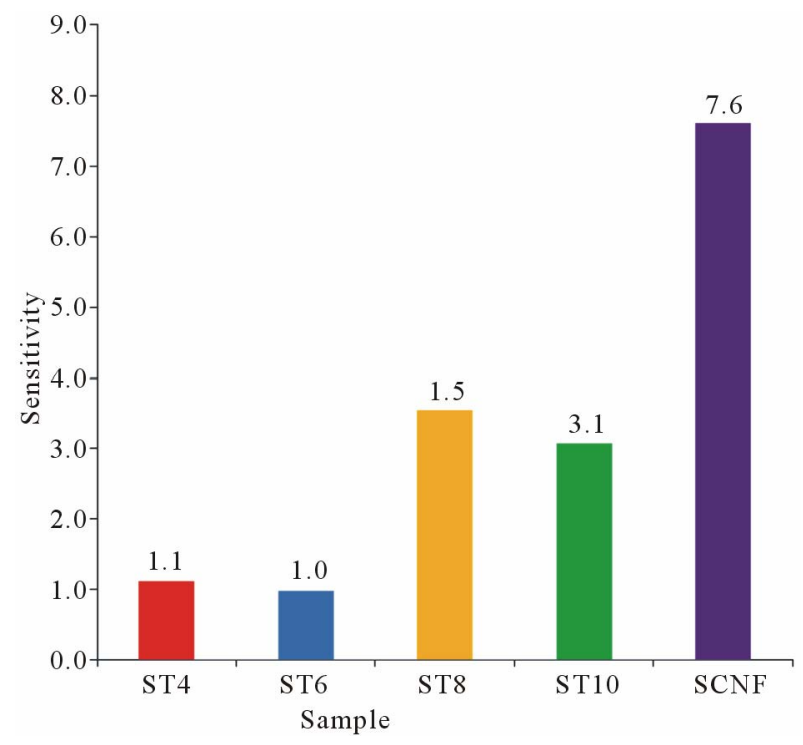

Figure 4. Sensitivity of various sensors to $50 \mu \mathrm{L}$ ethyl alcohol vapors displayed in bar chart.

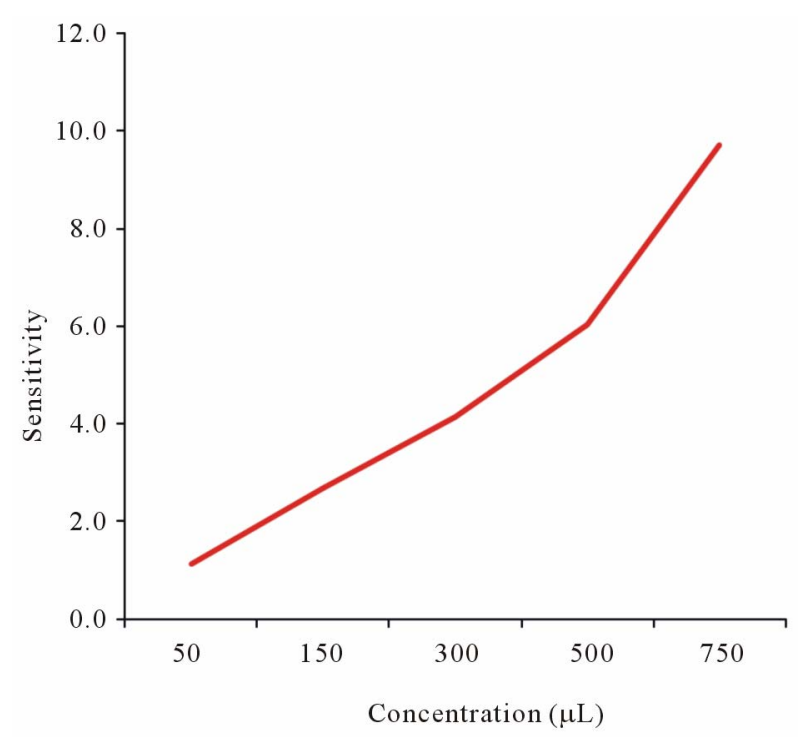

Figure 5. Senstivity of ST8 sensor to ethyl alcohol concentarion levels using static method. face. An increase in vapor concentration raises the surface coverage and thus increase in sensitivity is observed. Figure 7 shows the response time of $50 \mu \mathrm{L}$ ethyl alcohol vapors. Based on the results, SCNF sensor shows the outstanding sensitivity and response time.

To propose mechanisms for detection of ethyl alcohol vapor, it is worth mentioning that $\mathrm{SnO}_{2}$ has non-stoichiometric structures, free electrons originating from oxygen vacancies contribute to conductivity (n-type) by following equation:

$$
\mathrm{O}_{\mathrm{O}}(\text { bulk })=\mathrm{V}_{\mathrm{O}}+2 \mathrm{e}^{-}+1 / 2 \mathrm{O}_{2} \text { (gas) }
$$

Thus in an n-type metal-oxide semiconductor, conduction electrons $\left(\mathrm{e}^{-}\right)$come primarily from point defects

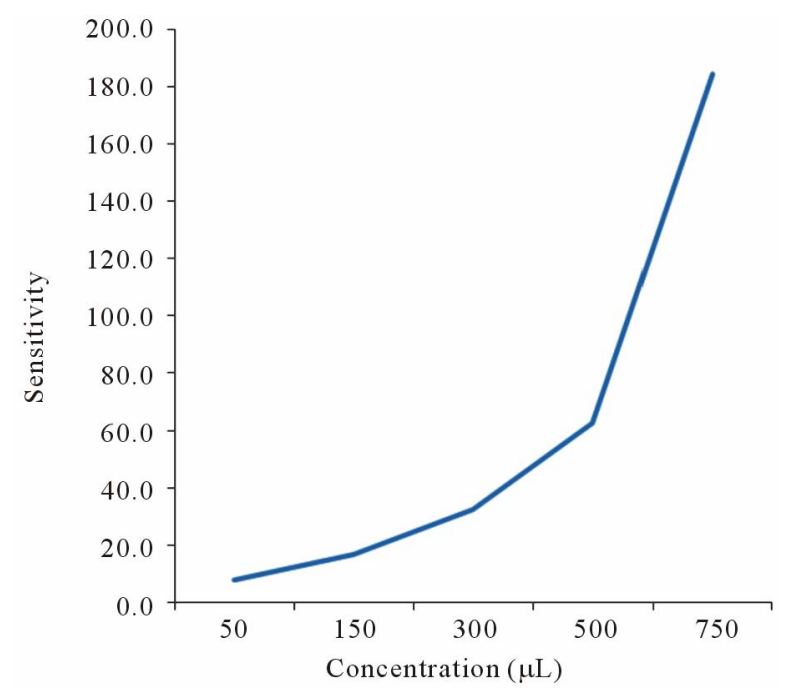

Figure 6. Senstivity of SCNF sensor to ethyl alcohol concentarion levels using static method.

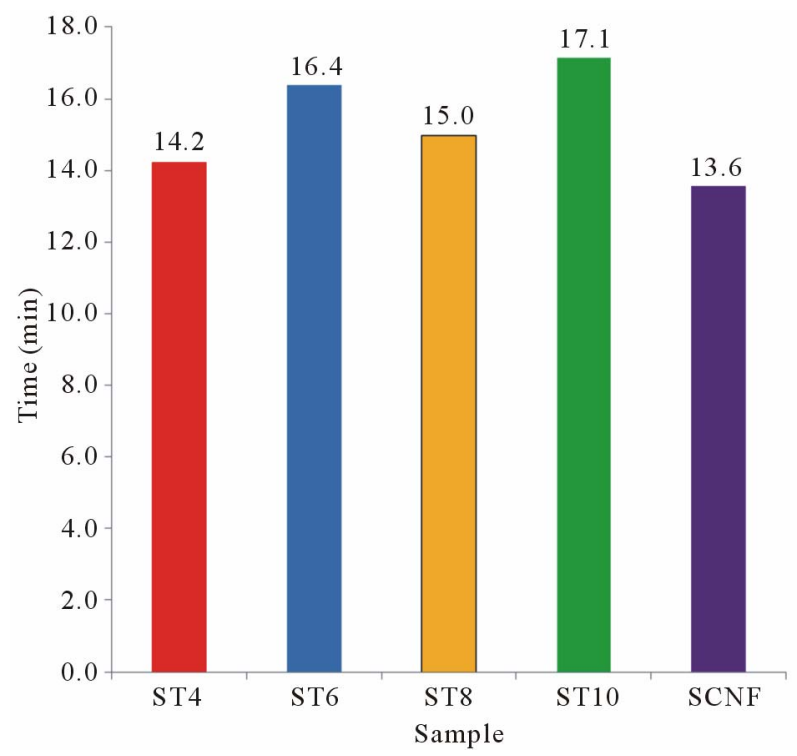

Figure 7. Response time (62.3\%) of various sensors under $50 \mu \mathrm{L}$ of ethyl alcohol. 
(oxygen vacancies and interstitial tin atoms) and play a major role in the gas sensing operation $[1,2,15]$, which is the combination of two adsorption reactions. So, the reaction sequences on ethyl alcohol, $\left(\mathrm{CH}_{3}\right) \mathrm{CH}_{2} \mathrm{OH}$, can be written in the following reaction steps with physical and chemical adsorption:

Step I for sensitizing reaction:

$$
\mathrm{O}_{2} \text { (gas) }+2 \mathrm{e}^{-} \rightarrow 2 \mathrm{O}^{-}+\Delta \mathrm{H}_{1}
$$

Step II for detection reaction:

$$
\begin{aligned}
& \left(\mathrm{CH}_{3}\right) \mathrm{CH}_{3} \mathrm{OH}+\mathrm{O}^{-}+\Delta \mathrm{H}_{2} \\
& \rightarrow \mathrm{CH}_{2} \mathrm{COCH}_{2}+\mathrm{H}_{2} \mathrm{O}+\Delta \mathrm{H}_{3}
\end{aligned}
$$

Surface morphology of the films is an important issue, as the smaller the grain size, the larger the specific surface area, which results in the greater adsorption and higher sensitivity $[16,17]$. The mechanisms for increase in response in $\mathrm{CNF}-\mathrm{SnO}_{2}$ composite can be explained via formation of (n-oxide)/(p-CNF)/(n-oxide) hetero-junction. When tin oxide (n-oxide) is exposed to ethyl alcohol gas, the gas molecules will react with oxygen ions previously adsorbed on the surface as depicted in step II releasing electrons, which increases the conductivity of sensing material. In the case of SCNF, the adsorption of the ethyl alcohol gas may change two depletions layers between $\mathrm{n}-\mathrm{p}$ and $\mathrm{p}-\mathrm{n}$ junctions as shown in Figure 8 with improved sensitivity. It is worth mentioning that n-type tin oxide and p-type CNF form a hetero-structure like the working principle of an n-p-n amplifier, CNF acts like a base, blocked electrons transfer from $\mathrm{n}$ (emitter) to $\mathrm{p}$ (collector), and thus lowering the barrier a little bit allows a large amount of electrons to pass from emitter to the collector [11]. Thus, this amplification effect can explain the improvement in gas sensor performance as compared with other virgin tin oxide samples investigated.

In order to seek an explanation for changes in sensor response of ST4, ST6, ST8 and ST10 sensors investigated, pore size distributions have to be measured. Decrease of pores is likely to be responsible for decrease of sensor response [18]. Highly porous sensors have high sensitivity [19]. The observed changes in the sensitivity of sensor elements may be attributed to porosity and particle size variation. Further detailed study is in progress to conclude the possible response mechanisms.

\section{Conclusion}

Gas sensors were prepared and characterized. It was ex-

\begin{tabular}{|c|c|c|}
\hline $\begin{array}{c}\mathrm{SnO}_{2} \\
\text { (n-type) }\end{array}$ & $\begin{array}{c}\mathrm{CNF} \\
\text { (p-type) }\end{array}$ & $\begin{array}{c}\mathrm{SnO}_{2} \\
(\text { n-type })\end{array}$ \\
\hline
\end{tabular}

Figure 8. Potential junctions to electronic conduction at grain boundaries for $\mathrm{SnO}_{2} / \mathrm{CNF} / \mathrm{SnO}_{2}$ materials analogous to n-p-n transistor amplifier. perimentally demonstrated that the tin oxide reinforced with carbon nanofibers improved the gas-sensing properties compared to tin oxide sensors investigated for ethyl alcohol, and produces a shorter response time. The gassensing mechanism of nano-composite sensor has been discussed. However, further study is needed via atomic force microscopy to understand higher sensitivity of 8 ton pressed and CNF doped sensors.

\section{Acknowledgements}

The authors gratefully acknowledge the support through NSF RISE grant \# HRD-0927644 and DHS grant 2010ST-062-000034. Authors would like to thank Drs. R. B. Lal and M. D. Aggarwal for keen interest in our research.

\section{REFERENCES}

[1] K. Ihokura and J. Watson, "The Stannic Oxide Gas Sensors," CRC Press, Boca Raton, 1994.

[2] G. Sberveglieri, "Gas Sensors: Principles, Operation and Developments," Kluwer Academic Publishers, London, 1992.

[3] S. W. Lee, P. P. Tsai and H. Chen, "Comparison Study of $\mathrm{SnO}_{2}$ Thin- and Thick-Film Gas Sensors," Sensors and Actuators B, Vol. 67, No. 1-2, 2000, pp. 122-127. doi:10.1016/S0925-4005(00)00390-7

[4] Th. Becker, S. Ahlers, C. B. Braunmuhl, G. Mulle and O. Kiesewetter, "Gas Sensing Properties of Thin- and ThickFilm Tin Oxide Materials," Sensors and Actuators B, Vol. 77, No. 1-2, 2001, pp. 55-61. doi:10.1016/S0925-4005(01)00672-4

[5] V. Guidi, et al., "Gas Sensing through Thick Film Technology," Sensors and Actuators B, Vol. 84, No. 1, 2002, pp. 72-77. doi:10.1016/S0925-4005(01)01077-2

[6] B. P. L. Costello, R. J. Ewen, N. Guernion and N. M. Ractlife, "Highly Sensitive Mixed Oxide Sensors for the Detection of Ethanol," Sensors and Actuators B, Vol. 87, No. 1, 2002, pp. 207-210. doi:10.1016/S0925-4005(02)00220-4

[7] N. Choi, C. Shim, K. Song, D. Lee, J. Huh and D. Lee, "Classification of Workplace Gases Using Temperature Modulation of Two $\mathrm{SnO}_{2}$ Sensing Films on Substrate," Sensors and Actuators B, Vol. 86, No. 2-3, 2002, pp. 251258. doi:10.1016/S0925-4005(02)00196-X

[8] K.-W. Kima, P.-S. Choa, S.-J. Kima, J.-H. Lee, C.-Y. Kangb, J.-S. Kimb and S.-J. Yoon, "The Selective Detection of $\mathrm{C}_{2} \mathrm{H}_{5} \mathrm{OH}$ Using $\mathrm{SnO}_{2}-\mathrm{ZnO}$ Thin Film Gas Sensors Prepared by Combinatorial Solution Deposition," Sensors and Actuators B, Vol. 123, No. 1, 2007, pp. 318324. doi:10.1016/j.snb.2006.08.028

[9] R. Leghrib, et al., "Gas Sensors Based on Multiwall Carbon Nanotubes Decorated with Tin Oxide Nanoclusters," Sensors and Actuators B, Vol. 145, No. 1, 2008, pp. 411416. doi:10.1016/j.snb.2009.12.044

[10] J. Gong, J. Sun and Q. Chen, "Micromachined Sol-Gel Carbon Nanotubes/ $\mathrm{SnO}_{2}$ Nanocomposite Hydrogen Sen- 
sor", Sensors and Actuators B, Vol. 130, No. 2, 2008, pp. 829-835. doi:10.1016/j.snb.2007.10.051

[11] N. V. Duy , N. V. Hieu, P. T. Huy and N. D. Chien, "Mixed $\mathrm{SnO}_{2} / \mathrm{TiO}_{2}$ Included with Carbon Nanotubes for Gas-Sensing Application," Physica E, Vol. 41, No. 2, 2008, pp. 258-263. doi:10.1016/j.physe.2008.07.007

[12] N. V. Hieu, N. A. P Duc, T. Trung, M. A. Tuan and N. C. Chien, "Gas-Sensing Properties of Tin Oxide Doped with Metal Oxide and Carbon Nanotubes: A Competitive Sensor for Ethanol and Liquid Petroleum," Sensors and Actuators B, Vol. 144, No. 2, 2010, pp. 450-456. doi:10.1016/j.snb.2009.03.043

[13] J. H. Yu and G. M. Choi, "Electrical and CO Gas Sensing Properties of $\mathrm{ZnO}-\mathrm{SnO}_{2}$ Composites," Sensors and Actuators B, Vol. 52, No. 3, 1998, pp. 251-256. doi:10.1016/S0925-4005(98)00275-5

[14] J. R. Currie, "Studies of Metal Oxide Chemical Sensors for the Detection of Volatile Organic Compounds," Ph.D. Thesis, AL A\&M University, Normal, 2008.

[15] H. Ogawa, A. Abe, A. M. Nishikawa and S. Hayakawa,
"Electrical Properties of Tin Oxide Ultrafine Particle Films," ECS Journal of Solid State Science and Technology, Vol. 28, 1985, p. 2020.

[16] J. Xu , Q. Pan, Y. A. Shun and Z. Tian, "Grain Size Control and Gas Sensing Properties of Tin Oxide," Sensors and Actuators B, Vol. 66, No. 1-3, 2000, pp. 277-279. doi:10.1016/S0925-4005(00)00381-6

[17] K. Arshak and I. Gaidan, "Development of a Novel Gas Sensor Based on Oxide Thick Films," Materials Science and Engineering: B, Vol. 118, No. 1-3, 2005, pp. 44-49. doi:10.1016/j.mseb.2004.12.061

[18] M. Kugishima, K. Shimanoe and N. Yamazoe, " $\mathrm{C}_{2} \mathrm{H}_{4} \mathrm{O}$ Sensing Properties of Thick-Film Sensor Using $\mathrm{La}_{2} \mathrm{O}_{3}$ Modified $\mathrm{SnO}_{2}$," Sensors and Actuators B, Vol. 118, No. 1-2, 2006, pp. 171-176. doi:10.1016/j.snb.2006.04.053

[19] S. S. Park and J. D. Mackenzie, "Thickness and Microstructure Effects of Alcohol Sensing of Tin Oxide Thin Films," Thin Solid Films, Vol. 274, No. 1-2, 1996, pp. 154-159. doi:10.1016/0040-6090(95)07075-3 